Indian J. Pure Appl. Math., 51(2): 689-703, June 2020

(c) Indian National Science Academy

DOI: $10.1007 / \mathrm{s} 13226-020-0424-6$

\title{
CLASSICAL ORTHOGONAL POLYNOMIALS VIA A SECOND-ORDER LINEAR DIFFERENTIAL OPERATORS
}

\author{
Baghdadi Aloui* and Wathek Chammam**** \\ *Department of Mathematics, College of Science Al-Zulfi, Majmaah University, \\ P.O.Box 66, Al-Majmaah 11952, Saudi Arabia \\ ** Department of Electro Mechanics, Higher Institute of Industrial Systems of \\ Gabès, Gabès University, Street Salah Eddine Elayoubi 6033 Gabès, Tunisia \\ e-mails:Baghdadi.Aloui@fsg.rnu.tn;w.chammam@mu.edu.sa
}

(Received 18 June 2018; after final revision 7 November 2018;

accepted 1 April 2019)

Let $T_{c}:=D(x-c)((x-c) D+2 \mathbb{I})$ be a second-order linear differential operator, where $c$ is an arbitrary complex number, $D:=\frac{d}{d x}$ and $\mathbb{I}$ represents the identity on the linear space of polynomials with complex coefficients. The aim of this paper is to describe all of the $T_{c}$-classical orthogonal polynomials. Two canonical situations appear: the Laguerre $\left\{L_{n}^{(2)}\right\}_{n \geq 0}$ and the Jacobi $\left\{P_{n}^{(\alpha-2,2)}\right\}_{n \geq 0}$

Key words : Orthogonal polynomials; quasi-definite linear functionals; classical polynomials, differential operators; structure relations.

2010 Mathematics Subject Classification : 33C45, 42C05.

\section{INTRODUCTION}

Classical orthogonal polynomials can be characterized by means of several properties [5, 8, 24]. Among them, the so-called Hahn's characterization states that $\left\{P_{n}(x)\right\}_{n \geq 0}$ is a sequence of classical orthogonal polynomials if and only if the sequence of its derivatives $\left\{P_{n}^{\prime}(x)\right\}_{n \geq 0}$ is also orthogonal. For classical orthogonal polynomials this is simple since for monic Hermite polynomials we have that $\frac{d}{d x} H_{n}(x)=n H_{n-1}(x)$ is again orthogonal; for monic Laguerre polynomials we have that $\frac{d}{d x} L_{n}^{(\alpha)}(x)=n L_{n-1}^{(\alpha+1)}(x)$ is also orthogonal; and for monic Jacobi polynomials $\frac{d}{d x} P_{n}^{(\alpha, \beta)}(x)=$ $n P_{n-1}^{(\alpha+1, \beta+1)}(x)$ is again orthogonal. Even in the case of orthogonality with respect to nonpositive 
weight functions we have that for Bessel polynomials $[13,14,25] \frac{d}{d x} B_{n}^{(\alpha)}(x)=n B_{n-1}^{(\alpha-2)}(x)$. In what follows we shall refer to this class as $D$-classical orthogonal polynomials where $D=\frac{d}{d x}$.

The concept of $O$-classical orthogonal polynomials, where $O$ is an operator on the space of polynomials, has been studied by many authors in the literature (see $[1-4,7,9,15-18,20]$ ).

Let $T_{c}$ be the following second-order linear differential operator

$$
T_{c}:=D(x-c)((x-c) D+2 \mathbb{I})
$$

where $\mathbb{I}$ denotes the identity operator and $D$ the classical derivative, as already mentioned.

The aim of this contribution is to find the sequences of monic orthogonal polynomials $\left\{P_{n}\right\}_{n \geq 0}$ such that the sequence of monic polynomials $\left\{Q_{n}\right\}_{n \geq 0}$ where $Q_{n}(x):=\frac{T_{c} P_{n}(x)}{(n+1)(n+2)}$, is also orthogonal. This constitutes the so-called Hahn's characterization of $T_{c}$-classical orthogonal polynomials.

Indeed, we prove that such polynomials are a subfamily of the well-known $D$-classical orthogonal polynomials (i.e. Hermite, Laguerre, Bessel and Jacobi). More precisely, we have as solution only the Laguerre orthogonal polynomials with $\alpha=2$ when $c=0$ and the Jacobi polynomials with parameters $(\alpha-2,2)$ when $c=1$.

The techniques that are applied here are similar to those used in $[2,3]$, where the authors study the Hahn's problem with respect to some differential operators that reduce by exactly one the degree of any polynomial, although the operator $T_{c}$ preserves the degree of any polynomial. Note that the operator used in reference [3], therein called $\mathscr{O}_{c ; 1,3,2}$, and the operator defined above are connected by the standard derivative, as follows:

$$
\mathscr{O}_{c ; 1,3,2}=D T_{c}
$$

Then, by recalling the equalities (2.22) and (2.23) of page 149 of Chihara's book [12], which asserts well-known differential identities fulfilled by the Laguerre and the Jacobi sequences, we can conclude that the work now gives reference [3].

The rest of this paper is organised as follows. In Section 2, we develop the terminology and basic definitions that will be used later on. In Section 3, we exhaustively describe the $T_{c}$-classical sequences.

\section{PRELIMINARiES AND Notations}

Let $\mathscr{P}$ be the linear space of polynomials in one variable with complex coefficients. The algebraic dual space of $\mathscr{P}$ will be represented by $\mathscr{P}^{\prime}$. We denote by $\langle u, p\rangle$ the action of $u \in \mathscr{P}^{\prime}$ on $p \in \mathscr{P}$ and 
by $(u)_{n}:=\left\langle u, x^{n}\right\rangle, n \geq 0$, the sequence of moments of $u$ with respect to the polynomial sequence $\left\{x^{n}\right\}_{n \geq 0}$.

Let us define the following operations in $\mathscr{P}^{\prime}$. For linear functional $u$, any polynomial $q$, and any $(a, b, c) \in \mathbb{C} \backslash\{0\} \times \mathbb{C}^{2}$, let $D u=u^{\prime}, q u,(x-c)^{-1} u, \tau_{-b} u$ and $h_{a} u$ be the linear functionals defined by duality, [21].

$$
\begin{aligned}
& \langle q u, p\rangle:=\langle u, q p\rangle, \\
& \left\langle u^{\prime}, p\right\rangle:=-\left\langle u, p^{\prime}\right\rangle, \\
& \left\langle(x-c)^{-1} u, p\right\rangle:=\left\langle u, \theta_{c} p\right\rangle, \quad \text { where } \theta_{c} p(x)=\frac{p(x)-p(c)}{x-c}, \\
& \left\langle\tau_{-b} u, p\right\rangle:=\left\langle u, \tau_{b} p\right\rangle, \quad \text { where } \quad \tau_{b} p(x)=p(x-b), \\
& \left\langle h_{a} u, p\right\rangle:=\left\langle u, h_{a} p\right\rangle, \quad \text { where } \quad h_{a} p(x)=p(a x), \quad \text { for every } p \in \mathscr{P} .
\end{aligned}
$$

A linear functional $u$ is called normalized if it satisfies $(u)_{0}=1$.

Let $\left\{P_{n}\right\}_{n \geq 0}$ be a infinite sequence of monic polynomials (SMP) with $\operatorname{deg} P_{n}=n$ and let $\left\{u_{n}\right\}_{n \geq 0}$ be its dual sequence, $u_{n} \in \mathbb{P}^{\prime}$, defined by $\left\langle u_{n}, P_{m}\right\rangle=\delta_{n, m}, n, m \geq 0$. Notice that $u_{0}$ is said to be the canonical functional associated with the $\operatorname{SMP}\left\{P_{n}\right\}_{n \geq 0}$. Recall that any $u \in \mathscr{P}^{\prime}$ can be represented as $u=\sum_{n=0}^{+\infty}\left\langle u, P_{n}\right\rangle u_{n}$. So, if $\left\{u_{n}^{[1]}\right\}_{n \geq 0}$ denotes the dual sequence of the SMP $\left\{P_{n}^{[1]}\right\}_{n \geq 0}$ where $P_{n}^{[1]}(x):=(n+1)^{-1} P_{n+1}^{\prime}(x), n \geq 0$, then $D u_{n}^{[1]}=-(n+1) u_{n+1}, n \geq 0$ [22]. Likewise, the dual sequence $\left\{\tilde{u}_{n}\right\}_{n \geq 0}$ of the shifted SMP $\left\{\tilde{P}_{n}\right\}_{n \geq 0}$, where $\tilde{P}_{n}(x):=a^{-n} P_{n}(a x+b)$ with $(a, b) \in \mathbb{C} \backslash\{0\} \times \mathbb{C}$, is given by $\tilde{u}_{n}=a^{n}\left(h_{a^{-1}} \circ \tau_{-b}\right) u_{n}, n \geq 0$ [22].

Let us recall that a form $u$ (linear functional) is said to be quasi-definite (regular) if there exists a unique sequence of monic polynomials $\left\{P_{n}\right\}_{n \geq 0}$, such that [21,22]

$$
\left\langle u, P_{n} P_{m}\right\rangle=r_{n} \delta_{n, m}, \quad n, m \geq 0, \quad r_{n} \neq 0, \quad n \geq 0
$$

The sequence $\left\{P_{n}\right\}_{n \geq 0}$ is said to be the sequence of monic orthogonal polynomials (SMOP) with respect to $u$. Note that $u=(u)_{0} u_{0}$, with $(u)_{0} \neq 0$. For any quasi-definite linear functional $u$ and any polynomial $\varphi$ such that $\varphi u=0$, it is then straightforward to prove that $\varphi=0$, [22].

Lemma 2.1 - [21]. The SMP $\left\{P_{n}\right\}_{n \geq 0}$, with dual sequence $\left\{u_{n}\right\}_{n \geq 0}$, is orthogonal with respect to $u_{0}$ if and only if one of the following statements hold:

(i) $u_{n}=\left\langle u_{0}, P_{n}^{2}\right\rangle^{-1} P_{n} u_{0}, n \geq 0$. 
(ii) $\left\{P_{n}\right\}_{n \geq 0}$ satisfies a Three-Term Recurrence Relation

$$
(\text { TTRR })\left\{\begin{array}{l}
P_{0}(x)=1, \quad P_{1}(x)=x-\beta_{0}, \\
P_{n+2}(x)=\left(x-\beta_{n+1}\right) P_{n+1}(x)-\gamma_{n+1} P_{n}(x), n \geq 0,
\end{array}\right.
$$

where $\beta_{n}=\left\langle u_{0}, x P_{n}^{2}\right\rangle /\left\langle u_{0}, P_{n}^{2}\right\rangle \in \mathbb{C}$ and $\gamma_{n+1}=\left\langle u_{0}, P_{n+1}^{2}\right\rangle /\left\langle u_{0}, P_{n}^{2}\right\rangle \in \mathbb{C} \backslash\{0\}$.

A linear functional $u$ is said to be positive-definite if it is quasi-definite i.e. it satisfies (1) and $r_{n}>0$ for every nonnegative integer $n$ (see [21]). Note that a linear functional $u$ is quasi-definite but it is not necessarily positive-definite.

The orthogonality is preserved by a shifting in the variable. Indeed, for the shifted sequence $\left\{\tilde{P}_{n}\right\}_{n \geq 0}$ where $\tilde{P}_{n}(x):=a^{-n} P_{n}(a x+b)$ with $(a, b) \in \mathbb{C} \backslash\{0\} \times \mathbb{C}$, the following Three-Term Recurrence Relation holds (see [12, 21])

$$
(\text { TTRR })\left\{\begin{array}{l}
\tilde{P}_{0}(x)=1, \quad \tilde{P}_{1}(x)=x-\tilde{\beta}_{0}, \\
\tilde{P}_{n+2}(x)=\left(x-\tilde{\beta}_{n+1}\right) \tilde{P}_{n+1}(x)-\tilde{\gamma}_{n+1} \tilde{P}_{n}(x), n \geq 0
\end{array}\right.
$$

where $\tilde{\beta}_{n}=a^{-1}\left(\beta_{n}-b\right)$ and $\tilde{\gamma}_{n+1}=a^{-2} \gamma_{n+1}$.

Notice that $\left\{\tilde{P}_{n}\right\}_{n \geq 0}$ is orthogonal with respect to the linear functional $\tilde{u}_{0}=\left(h_{a^{-1}} \circ \tau_{-b}\right) u_{0}$.

A linear functional $u$ is said to be $D$-classical when it is quasi-definite and there exist two polynomials $\Phi$ and $\Psi, \Phi$ monic, $\operatorname{deg} \Phi=t \leq 2$, and $\operatorname{deg} \Psi=1$, such that $u$ satisfies a Pearson's equation (see [11, 21-23])

$$
\text { (PE) }(\Phi u)^{\prime}+\Psi u=0
$$

In such a case, the corresponding SMOP $\left\{P_{n}\right\}_{n \geq 0}$ is said to be $D$-classical.

Any shift leaves invariant the $D$-classical character. Indeed, the shifted linear functional $\tilde{u}=$ $\left(h_{a^{-1}} \circ \tau_{-b}\right) u$ fulfils [22]

$$
(\tilde{\Phi} \tilde{u})^{\prime}+\tilde{\Psi} \tilde{u}=0
$$

where $\tilde{\Phi}(x)=a^{-t} \Phi(a x+b)$ and $\tilde{\Psi}(x)=a^{1-t} \Psi(a x+b)$.

It is well-known that any $D$-classical polynomial sequence $\left\{P_{n}\right\}_{n \geq 0}$ can be characterized by taking into account its orthogonality and a First Structure Relation (FSR) or a Second Structure Relation (SSR) as follows, [6, 11-13, 22]:

$$
\begin{array}{ll}
(\mathrm{FSR}) & \Phi(x) P_{n+1}^{\prime}(x)=r(x ; n) P_{n+1}(x)+s_{n} P_{n}(x), n \geq 0 \\
(\mathrm{SSR}) & P_{n}(x)=P_{n}^{[1]}(x)+a_{n} P_{n-1}^{[1]}(x)+b_{n} P_{n-2}^{[1]}(x), n \geq 0
\end{array}
$$


The $D$-classical orthogonal polynomials are essentially the only polynomial (not just orthogonal polynomial) systems that satisfy a Second-Order Differential Equation (SODE, in short), Bochner [10], (see also [5, 23]), of the form

$$
\Phi(x) P_{n+1}^{\prime \prime}(x)-\Psi(x) P_{n+1}^{\prime}(x)=\omega_{n} P_{n+1}(x), n \geq 0,
$$

with $\operatorname{deg} \phi \leq 2, \operatorname{deg} \psi=1$ and where $(n+1)\left(\frac{1}{2} \phi^{\prime \prime}(0) n+\psi^{\prime}(0)\right)=\omega_{n} \neq 0, n \geq 0$. For the four canonical situations (three positive-definite cases, namely Hermite, Laguerre, and Jacobi, and one quasi-definite case, namely Bessel), in the next table we summarize the parameters involved in (2)-(6), (for more details, see [5, 11, 21-23]).

Table: Some basic characteristics of classical orthogonal polynomials.

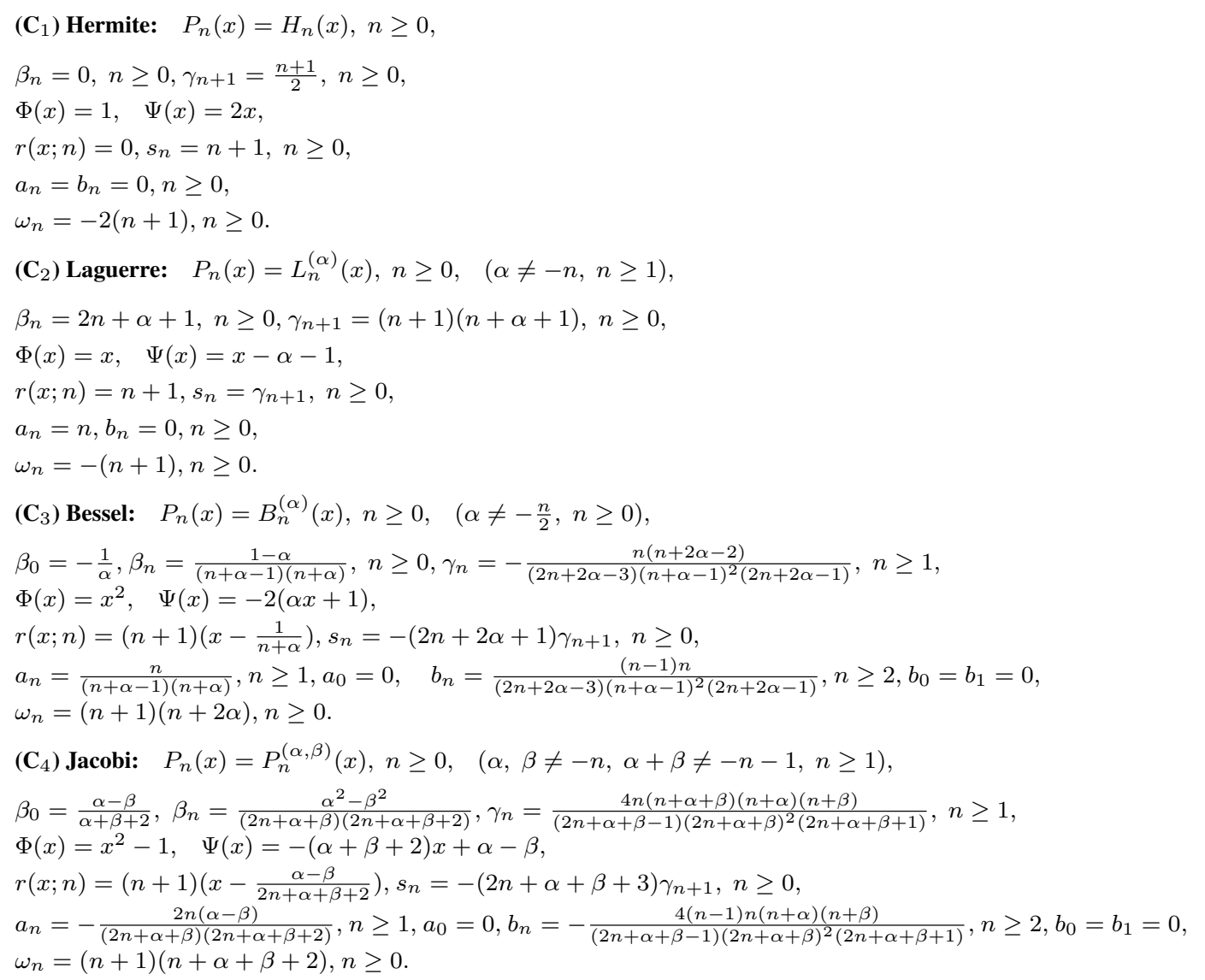

Notice that a linear functional $D$-classical is not necessarily positive-definite. 
Now, recall that the latter operator is given by

$$
\begin{aligned}
T_{c}: \mathscr{P} & \longrightarrow \mathscr{P} \\
f & \longmapsto(x-c)^{2} f^{\prime \prime}+4(x-c) f^{\prime}+2 f,
\end{aligned}
$$

It is clear that $T_{c}$ preserves the degree of any polynomial. We have

$$
T_{c}(x-c)^{n}=(n+1)(n+2)(x-c)^{n}, n \geq 0 .
$$

By transposition of the operator $T_{c}$, we get

$$
{ }^{t} T_{c}=(x-c)^{2} D^{2}
$$

For any SMP $\left\{P_{n}\right\}_{n \geq 0}$, we define

$$
Q_{n}(x):=\frac{T_{c} P_{n}(x)}{(n+1)(n+2)}, \quad n \geq 0 .
$$

Clearly, $\left\{Q_{n}\right\}_{n \geq 0}$ is a SMP and $\operatorname{deg} Q_{n}=n$. If $\left\{v_{n}\right\}_{n \geq 0}$ denotes the dual sequence of $\left\{Q_{n}\right\}_{n \geq 0}$, then we have

$$
(x-c)^{2} v_{n}^{\prime \prime}=(n+1)(n+2) u_{n}, \quad n \geq 0 .
$$

Note that for $c=0$ and using the representation of Laguerre polynomials in terms of hypergeometric series [19]:

$$
L_{n}^{(\alpha)}(x)=\sum_{\nu=0}^{n}(-1)^{n-\nu}\left(\begin{array}{l}
n \\
\nu
\end{array}\right) \frac{\Gamma(n+\alpha+1)}{\Gamma(\nu+\alpha+1)} x^{\nu}
$$

with $\alpha=2$, we obtain

$$
\left(x^{2} D^{2}+4 x D+2 \mathbb{I}\right) L_{n}^{(2)}(x)=(n+1)(n+2) L_{n}^{(0)}(x), \quad n \geq 0 .
$$

Equivalently

$$
L_{n}^{(0)}(x)=\frac{T L_{n}^{(2)}(x)}{(n+1)(n+2)}, n \geq 0,
$$

where $T:=x^{2} D^{2}+4 x D+2 \mathbb{I}$. This implies that $Q_{n}(x)=L_{n}^{(0)}(x), n \geq 0$, which is an example of solution of our problem. The goal of this manuscript is to describe all of the sequences of $T_{c}$-classical orthogonal polynomials in the Hahn's sense, where $T_{c}, c \in \mathbb{C}$ is the above operator. 


\section{The $T_{c}$-Classical Orthogonal Polynomials}

Definition 3.1 - Let $u_{0}$ be a quasi-definite linear functional and let $\left\{P_{n}\right\}_{n \geq 0}$ be the corresponding SMOP. We call $\left\{P_{n}\right\}_{n \geq 0}$ is $T_{c}$-classical if $\left\{T_{c} P_{n}\right\}_{n \geq 0}$ is also orthogonal. In this case, $u_{0}$ is also said to be an $T_{c}$-classical linear functional.

Any shift leaves invariant the $T_{c}$-classical character.

Lemma 3.1 - When $\left\{P_{n}\right\}_{n \geq 0}$ is $T_{c}$-classical, then for any $(a, b) \in \mathbb{C} \backslash\{0\} \times \mathbb{C}$ the shifted polynomial sequence $\left\{\tilde{P}_{n}\right\}_{n \geq 0}$ given by $\tilde{P}_{n}(x)=a^{-n} P_{n}(a x+b), n \geq 0$, is $T_{\tilde{c}}$-classical, where $\tilde{c}=a^{-1}(c-b)$.

Proof : Assume that $\left\{P_{n}\right\}_{n \geq 0}$ is $T_{c}$-classical. By Definition 3.1, the SMP $\left\{Q_{n}(; c)\right\}_{n \geq 0}$ given by (9) that is,

$$
(n+1)(n+2) Q_{n}(x)=(x-c)^{2} P_{n}^{\prime \prime}(x)+4(x-c) P_{n}^{\prime}(x)+2 P_{n}(x), n \geq 0
$$

is orthogonal.

For any fixed $(a, b) \in \mathbb{C} \backslash\{0\} \times \mathbb{C}$, let $\left\{\tilde{P}_{n}\right\}_{n \geq 0}$ and $\left\{\tilde{Q}_{n}\right\}_{n \geq 0}$ be the shifted SMOP given by $\tilde{P}_{n}(x)=a^{-n} P_{n}(a x+b)$ and $\tilde{Q}_{n}(x)=a^{-n} Q_{n}(a x+b)$. By replacing $x$ by $a x+b$ in (13), we get

$$
(n+1)(n+2) \tilde{Q}_{n}(x)=(x-\tilde{c})^{2} \tilde{P}_{n}^{\prime \prime}(x)+4(x-\tilde{c}) \tilde{P}_{n}^{\prime}(x)+2 \tilde{P}_{n}(x), n \geq 0
$$

where $\tilde{c}=a^{-1}(c-b)$.

Equivalently $\tilde{Q}_{n}(x)=\frac{T_{\tilde{c}} \tilde{P}_{n}(x)}{(n+1)(n+2)}, n \geq 0$. Hence, $\left\{\tilde{P}_{n}\right\}_{n \geq 0}$ is $T_{\tilde{c} \text {-classical. }}$

Our next goal is to describe all of the the $T_{c}$-classical polynomial sequences. Assume that $\left\{P_{n}\right\}_{n \geq 0}$ and $\left\{Q_{n}\right\}_{n \geq 0}$ are SMOP satisfying

$$
\begin{aligned}
& \left\{\begin{array}{l}
P_{0}(x)=1, P_{1}(x)=x-\beta_{0}, \\
P_{n+2}(x)=\left(x-\beta_{n+1}\right) P_{n+1}(x)-\gamma_{n+1} P_{n}(x), \gamma_{n+1} \neq 0, n \geq 0,
\end{array}\right. \\
& \left\{\begin{array}{l}
Q_{0}(x)=1, Q_{1}(x)=x-\xi_{0}, \\
Q_{n+2}(x)=\left(x-\xi_{n+1}\right) Q_{n+1}(x)-\lambda_{n+1} Q_{n}(x), \lambda_{n+1} \neq 0, n \geq 0 .
\end{array}\right.
\end{aligned}
$$

The dual sequences of $\left\{P_{n}\right\}_{n \geq 0}$ and $\left\{Q_{n}\right\}_{n \geq 0}$ will be denoted by $\left\{u_{n}\right\}_{n \geq 0}$ and $\left\{v_{n}\right\}_{n \geq 0}$, respectively. By Lemma 2.1(i), we get

$$
u_{n}=\frac{P_{n}}{\left\langle u_{0}, P_{n}^{2}\right\rangle} u_{0}, n \geq 0 \quad ; \quad v_{n}=\frac{Q_{n}}{\left\langle v_{0}, Q_{n}^{2}\right\rangle} v_{0}, n \geq 0 .
$$

In the following result, we prove that the sequence $\left\{Q_{n}\right\}_{n \geq 0}$ is $D$-classical.

Lemma 3.2 - The following properties hold. 
(i) $(\mathrm{SSR}) \quad Q_{n}(x)=Q_{n}^{[1]}(x)+c_{n} Q_{n-1}^{[1]}(x)+d_{n} Q_{n-2}^{[1]}(x), n \geq 0$, where

$$
c_{n}=\frac{n}{2}\left(\beta_{n}-\xi_{n}\right), n \geq 0, \quad d_{n}=\frac{n-1}{2}\left(\frac{n}{n+2} \gamma_{n}-\lambda_{n}\right), n \geq 1, d_{0}=0 .
$$

(ii) (PE) $\left(\Phi v_{0}\right)^{\prime}+\Psi v_{0}=0$, where

$$
\begin{aligned}
\kappa \Phi(x) & =d_{2}\left(\lambda_{1} \lambda_{2}\right)^{-1} Q_{2}(x)+c_{1} \lambda_{1}^{-1} Q_{1}(x)+1, \\
\Psi(x) & =\left(\kappa \lambda_{1}\right)^{-1} Q_{1}(x) .(\kappa \text { is a normalization factor })
\end{aligned}
$$

PROOF : Let us introduce the sequence of monic polynomials $\left\{Z_{n}\right\}_{n \geq 0}$ given by

$$
(n+2) Z_{n+1}(x):=(x-c)^{2} P_{n}^{\prime}(x)+2(x-c) P_{n}(x), n \geq 0 .
$$

By taking derivatives in both hand sides of (13) and taking into account (17), we get

$$
Z_{n}^{[1]}(x)=Q_{n}(x), n \geq 0 .
$$

Notice that $Z_{n}(x)$ is a monic primitive of $Q_{n}(x)$.

From (14) and (17), we obtain

$$
\begin{gathered}
(n+4) Z_{n+3}(x)=(n+3)\left(x-\beta_{n+1}\right) Z_{n+2}(x)-(n+2) \gamma_{n+1} Z_{n+1}(x)+(x-c)^{2} P_{n+1}(x), n \geq 0, \\
3 Z_{2}(x)=2\left(x-\beta_{0}\right) Z_{1}(x)+(x-c)^{2} P_{0}(x) .
\end{gathered}
$$

By differentiating in both sides of the previous identities and inserting (18), we get

$$
\begin{aligned}
2(n+3) Z_{n+2}(x)= & (n+3)(n+4) Q_{n+2}(x)-(n+2)\left[(n+3)\left(x-\beta_{n+1}\right) Q_{n+1}(x)\right. \\
& \left.-(n+1) \gamma_{n+1} Q_{n}(x)\right], n \geq 0, \\
2 Z_{1}(x)= & 3 Q_{1}(x)-\left(x-\beta_{0}\right) Q_{0}(x) .
\end{aligned}
$$

Then, by (15), it follows that

$$
\begin{aligned}
Z_{n+2}(x) & =Q_{n+2}(x)+e_{n+1} Q_{n+1}(x)+f_{n} Q_{n}(x), n \geq 0 \\
Z_{1}(x) & =Q_{1}(x)+e_{0} Q_{0}(x) .
\end{aligned}
$$

where $e_{n}=\frac{n+1}{2}\left(\beta_{n}-\xi_{n}\right)$ and $f_{n}=\frac{n+2}{2}\left(\frac{n+1}{n+3} \gamma_{n+1}-\lambda_{n+1}\right)$.

By differentiating both hand sides of (19) and using (18), (i) holds. 
Let $\left\{v_{n}^{[1]}\right\}_{n \geq 0}$ be the dual sequence of $\left\{Q_{n}^{[1]}\right\}_{n \geq 0}$. From (i), we have $\left\langle v_{0}^{[1]}, Q_{n}\right\rangle=0, n \geq 3$, $\left\langle v_{0}^{[1]}, Q_{2}\right\rangle=d_{2},\left\langle v_{0}^{[1]}, Q_{1}\right\rangle=c_{1}$, and $\left\langle v_{0}^{[1]}, Q_{0}\right\rangle=1$. So, $v_{0}^{[1]}=d_{2} v_{2}+c_{1} v_{1}+v_{0}$, and by (16), we get $v_{0}^{[1]}=\kappa \Phi(x) v_{0}$, where $\kappa \Phi(x)=d_{2} \lambda_{1}^{-1} \lambda_{2}^{-1} Q_{2}(x)+c_{1} \lambda_{1}^{-1} Q_{1}(x)+1$ and $\kappa$ is a normalization factor. Because $\left(v_{0}^{[1]}\right)^{\prime}=-v_{1}=-\lambda_{1}^{-1} Q_{1} v_{0}$, then $\left(\Phi v_{0}\right)^{\prime}+\Psi v_{0}=0$, where $\Psi(x)=\left(\kappa \lambda_{1}\right)^{-1} Q_{1}(x)$. Hence, (ii) holds.

Lemma 3.3 - There are two non-zero polynomials $F$ and $G$, with $\operatorname{deg} F \leq 2$ and $\operatorname{deg} G \leq 1$, such that

(i) $(x-c)^{2} v_{0}=F(x) u_{0}$.

(ii) $F(x) Q_{n}^{\prime \prime}(x)+G(x) Q_{n}^{\prime}(x)+2 Q_{n}(x)=\rho_{n} P_{n}(x), n \geq 0$, where

$$
\begin{aligned}
F(x) & =\frac{1}{2}\left(\rho_{2} P_{2}(x)-G(x) Q_{2}^{\prime}(x)-2 Q_{2}(x)\right) \\
G(x) & =\rho_{1} P_{1}(x)-2 Q_{1}(x), \\
\rho_{n} & =(n+1)(n+2) \frac{\left\langle v_{0}, Q_{n}^{2}\right\rangle}{\left\langle u_{0}, P_{n}^{2}\right\rangle}=n(n-1) \frac{F^{\prime \prime}(0)}{2}+n G^{\prime}(0)+2 .
\end{aligned}
$$

(iii) The following relations hold

(a) $A(x) F(x)=2 \Phi^{2}(x)$,

(b) $\Phi(x) G(x)=-2\left(\Phi^{\prime}(x)+\Psi(x)\right) F(x)$,

where,

$$
A(x)=\left(2 \Phi^{\prime}(x)+\Psi(x)\right)\left(\Phi^{\prime}(x)+\Psi(x)\right)-\left(\Phi^{\prime \prime}(x)+\Psi^{\prime}(x)\right) \Phi(x) .
$$

PROOF : From (10) and (16), we obtain

$$
(x-c)^{2} Q_{n}(x) v_{0}^{\prime \prime}+2(x-c)^{2} Q_{n}^{\prime}(x) v_{0}^{\prime}+(x-c)^{2} Q_{n}^{\prime \prime}(x) v_{0}=\rho_{n} P_{n}(x) u_{0}, n \geq 0,
$$

where $\rho_{n}=(n+1)(n+2) \frac{\left\langle v_{0}, Q_{n}^{2}\right\rangle}{\left\langle u_{0}, P_{n}^{2}\right\rangle}$.

From (21) with $n=0$, we obtain

$$
(x-c)^{2} v_{0}^{\prime \prime}=2 u_{0}
$$

Using (21) and (23), it follows that

$$
(x-c)^{2} Q_{n}^{\prime \prime}(x) v_{0}+2(x-c)^{2} Q_{n}^{\prime}(x) v_{0}^{\prime}=\left(\rho_{n} P_{n}(x)-2 Q_{n}(x)\right) u_{0}
$$


For $n=1$, (23) becomes

$$
2(x-c)^{2} v_{0}^{\prime}=G(x) u_{0}
$$

where $G(x)=\rho_{1} P_{1}(x)-2 Q_{1}(x)$.

By inserting (24) in (23), we obtain

$$
(x-c)^{2} Q^{\prime \prime}(x)_{n} v_{0}=\left(\rho_{n} P_{n}(x)-2 Q_{n}(x)-G(x) Q^{\prime}(x)_{n}\right) u_{0} .
$$

Hence, taking $n=2$ in (25), (i) holds.

Meanwhile, by substituting $(x-c)^{2} v_{0}=F u_{0}$ in (25) and taking into account the quasi-definiteness of $u_{0}$, we deduce (ii).

By using Lemma 3.2(ii), we can write

$$
\Phi v_{0}^{\prime}=-\left(\Phi^{\prime}+\Psi\right) v_{0}, \quad \Phi^{2} v_{0}^{\prime \prime}=A v_{0},
$$

where $A=\left(2 \Phi^{\prime}+\Psi\right)\left(\Phi^{\prime}+\Psi\right)-\left(\Phi^{\prime \prime}+\Psi^{\prime}\right) \Phi$.

In contrast, if we multiply (22) by $\Phi^{2}$ and we take into account (26), (i) and also the quasidefiniteness of $u_{0}$, we get (iii) (a).

In the same way, multiplying both sides of (24) by $\Phi$ and using (26) (i), and then taking into account the quasi-definiteness of $u_{0}$, we deduce (iii) (b).

Now, we will describe all of the $T_{c}$-classical polynomial sequences.

Theorem 3.1 - The $T_{c}$-classical polynomial sequences are, up to a suitable affine transformation in the variable, one of the following D-classical polynomial sequences

(i) $P_{n}(x)=L_{n}^{(2)}(x)$ and $Q_{n}(x)=L_{n}^{(0)}(x), n \geq 0$, with $c=0$.

(ii) $P_{n}(x)=P_{n}^{(\alpha-2,2)}(x)$ and $Q_{n}(x)=P_{n}^{(\alpha, 0)}(x), n \geq 0$, with $c=1$ and where $\alpha \neq-n+2, n \geq$ 1.

ProOF : From Lemma 3.2, $\left\{Q_{n}\right\}_{n \geq 0}$ is $D$-classical. According to Lemma 3.1, we will analyze the following situations:

$\left(\mathbf{S}_{1}\right) \cdot\left\{Q_{n}\right\}_{n \geq 0}$ is the Hermite SMOP. From Table $\left(\mathbf{C}_{1}\right), A(x)=2\left(2 x^{2}-1\right)$. Then, from Lemma 3.3(iii) (a), we get $2\left(2 x^{2}-1\right) F(x)=2$. This yields a contradiction. 
$\left(\mathbf{S}_{2}\right) \cdot\left\{Q_{n}\right\}_{n \geq 0}$ is the Laguerre SMOP. From Table $\left(\mathbf{C}_{2}\right), A(x)=x^{2}-2 \alpha x+\alpha(\alpha-1)$. Therefore, from Lemma 3.3, (iii), we have

$$
\begin{aligned}
& \left(x^{2}-2 \alpha x+\alpha(\alpha-1)\right) F(x)=2 x^{2}, \\
& -2(x-\alpha) F(x)=x G(x) .
\end{aligned}
$$

From (27), we can deduce, $F(x)=2$ and $\alpha=0$. Hence, we get $A(x)=x^{2}$ and $G(x)=-4$. According to Lemma 3.3 (ii), we have $2 Q_{n}^{\prime \prime}(x)-4 Q_{n}^{\prime}(x)+2 Q_{n}(x)=\rho_{n} P_{n}(x), n \geq 0$. Thus, $\rho_{n}=2, n \geq 0$. Therefore,

$$
Q_{n}^{\prime \prime}(x)-2 Q_{n}^{\prime}(x)+Q_{n}(x)=P_{n}(x), \quad n \geq 0
$$

This gives, for $n=1, \beta_{0}=3$. Since $2=\rho_{1}=6\left\langle v_{0}, Q_{1}^{2}\right\rangle\left\langle u_{0}, P_{1}^{2}\right\rangle^{-1}=6 \lambda_{1} \gamma_{1}^{-1}$, we get $\gamma_{1}=3$. In contrast, we have by Lemma 3.2(i), $\beta_{n}=2 n+3, n \geq 1$ and $\gamma_{n}=n(n+2), n \geq 2$. Hence, we obtain $\beta_{n}=2 n+3$ and $\gamma_{n+1}=(n+1)(n+3), n \geq 0$. Then, $P_{n}(x)=L_{n}^{(2)}(x), n \geq 0$, with $Q_{n}(x)=L_{n}^{(0)}(x), n \geq 0$, and $(x-c)^{2} v_{0}=2 u_{0}$. Making $n=1$ in (13), we get $c=0$. Consequently, the following relations hold:

$$
\begin{aligned}
& L_{n}^{(0)}(x)=\frac{T L_{n}^{(2)}(x)}{(n+1)(n+2)}, n \geq 0, \\
& L_{n}^{(0)^{\prime \prime}}(x)-2 L_{n}^{(0)}(x)+L_{n}^{(0)}(x)=L_{n}^{(2)}(x), n \geq 0 .
\end{aligned}
$$

$\left(\mathbf{S}_{3}\right) .\left\{Q_{n}\right\}_{n \geq 0}$ is the Bessel SMOP with parameter $\alpha \neq-n / 2, n \geq 0$. In this case, we get $A(x)=2(1-\alpha)(3-2 \alpha) x^{2}+4(2 \alpha-3) x+4$. Using Lemma 3.3(iii) (a), we obtain $A(x) F(x)=2 x^{4}$. This requires that $\operatorname{deg} A=2$ and $\operatorname{deg} F=2$ because $\operatorname{deg} A \leq 2, \operatorname{deg} F \leq 2$, and $\operatorname{deg} A+\operatorname{deg} F=4$. However, from the previous equation, we must have $A(0)=0$, that contradicts the fact that $A(0)=4$.

$\left(\mathbf{S}_{4}\right) .\left\{Q_{n}\right\}_{n \geq 0}$ is the Jacobi SMOP with parameters $\alpha$ and $\beta$ satisfying $\alpha, \beta \neq-n, \alpha+\beta \neq$ $-n-1, n \geq 1$. Then, we have

$$
A(x)=(\alpha+\beta-1)\left((\alpha+\beta) x^{2}+2(\beta-\alpha) x\right)+(\alpha-\beta)^{2}-(\alpha+\beta) .
$$

By using Lemma 3.3(iii) (a), we obtain $A(x) F(x)=2\left(x^{2}-1\right)^{2}$. The fact that $\operatorname{deg} A+\operatorname{deg} F=4$, $\operatorname{deg} F \leq 2$ and $\operatorname{deg} A \leq 2$, yields $\operatorname{deg} A=2$ and $\operatorname{deg} F=2$. Meanwhile, the previous equation becomes $F(x)$ divides $\left(x^{2}-1\right)^{2}$, hence there are three situations to be considered. Either $F(x)=$ $\mu(x+1)^{2}, F(x)=\mu(x-1)^{2}$, or $F(x)=\mu(x-1)(x+1)$, where $\mu$ is a non-zero real number. 
$\left(\mathbf{S}_{4,1}\right) . F(x)=\mu(x+1)^{2}, \mu \neq 0$. According to Lemma 3.3(iii), we easily obtain

$$
\begin{aligned}
\mu A(x) & =2(x-1)^{2}, \\
(x-1) G(x) & =2 \mu((\alpha+\beta) x+\beta-\alpha) .
\end{aligned}
$$

From (31) and (32), we get

$$
\left\{\begin{array}{l}
(\alpha+\beta-1)(\alpha+\beta) \mu=2 \\
(\alpha+\beta-1)(\alpha-\beta) \mu=2, \\
\left((\alpha-\beta)^{2}-(\alpha+\beta)\right) \mu=2 .
\end{array}\right.
$$

Clearly, $\beta=0$ and then $\mu=\frac{2}{\alpha(\alpha-1)}$ with $\alpha(\alpha-1) \neq 0$. Hence, (32) and (33) gives

$$
\begin{aligned}
& A(x)=\alpha(\alpha-1)(x-1)^{2}, \\
& G(x)=\frac{4}{\alpha-1}(x+1) .
\end{aligned}
$$

Then, by virtue of Lemma 3.3(ii), we can deduce

$$
\frac{2}{\alpha(\alpha-1)}(x+1)^{2} Q_{n}^{\prime \prime}(x)+\frac{4}{\alpha-1}(x+1) Q_{n}^{\prime}(x)+2 Q_{n}(x)=\rho_{n} P_{n}(x), n \geq 0 .
$$

Thus $\rho_{n}=\frac{2 n(n-1)}{\alpha(\alpha-1)}+\frac{4 n}{\alpha-1}+2, n \geq 0$.

For $n=1$, we obtain $\beta_{0}=\frac{\alpha-4}{\alpha+2}$. Since $2 \frac{\alpha+1}{\alpha-1}=\rho_{1}=6\left\langle v_{0}, Q_{1}^{2}\right\rangle\left\langle u_{0}, P_{1}^{2}\right\rangle^{-1}=6 \lambda_{1} \gamma_{1}^{-1}$, we get $\gamma_{1}=\frac{12(\alpha-1)}{(\alpha+2)^{2}(\alpha+3)}$.

From Table

$\left(\mathbf{C}_{4}\right)$ and by Lemma 3.2(i), we finally get

$$
\begin{aligned}
\beta_{n} & =\frac{\alpha(\alpha-4)}{(2 n+\alpha)(2 n+\alpha+2)}, n \geq 0, \\
\gamma_{n+1} & =\frac{4(n+1)(n+3)(n+\alpha+1)(n+\alpha-1)}{(2 n+\alpha+1)(2 n+\alpha+2)^{2}(2 n+\alpha+3)}, n \geq 0 .
\end{aligned}
$$

Thus, we conclude that $P_{n}(x)=P_{n}^{(\alpha-2,2)}(x)$, and $Q_{n}(x)=P_{n}^{(\alpha, 0)}(x), n \geq 0$ with $\alpha \neq$ $-n+2, n \geq 1$. In addition, by (13) with $n=1$, (14) and (15), we get $c=\frac{1}{2}\left(3 \xi_{0}-\beta_{0}\right)=1$. By Lemma 3.3(i), $(x-1)^{2} v_{0}=\frac{2}{\alpha(\alpha-1)}(x+1)^{2} u_{0}$. Consequently,

$$
\begin{aligned}
& P_{n}^{(\alpha, 0)}(x)=\frac{T_{1} P_{n+1}^{(\alpha-2,2)}(x)}{(n+1)(n+2)}, \\
& (x+1)^{2} P_{n}^{(\alpha, 0)^{\prime \prime}}(x)+2 \alpha(x+1) P_{n}^{(\alpha, 0)^{\prime}}(x) \\
& \quad+\alpha(\alpha-1) P_{n}^{(\alpha, 0)}(x)=\chi_{n, \alpha} P_{n}^{(\alpha-2,2)}(x), n \geq 0,
\end{aligned}
$$


where $\chi_{n, \alpha}=(n+\alpha)(n+\alpha-1), n \geq 0$.

$\left(\mathbf{S}_{4,2}\right) . \quad F(x)=\mu(x-1)^{2}, \mu \neq 0$. By a similar computation as in $\left(\mathbf{S}_{4,1}\right)$, we get $P_{n}(x)=$ $P_{n}^{(2, \beta-2)}(x), n \geq 0$, where $\beta \neq-n+2, n \geq 1$, and also $Q_{n}(x)=P_{n}^{(0, \beta)}(x), n \geq 0$, and $c=-1$. Through a suitable shift, we get $\left(\mathbf{S}_{4,1}\right)$. Indeed, from Lemma 3.1 and taking into account $P_{n}^{(\alpha, \beta)}(-x)=(-1)^{n} P_{n}^{(\beta, \alpha)}(x)$ (see [12]), the SMOP $\left\{\tilde{P}_{n}\right\}_{n \geq 0}$ where $\tilde{P}_{n}(x)=(-1)^{n} P_{n}(-x)=$ $P_{n}^{(\beta-2,2)}(x)$, is $T_{1}$-classical.

$\left(\mathbf{S}_{4,3}\right) . F(x)=\mu(x-1)(x+1), \mu \neq 0$. According to Lemma 3.3(iii), we obtain

$$
\begin{aligned}
\mu A(x) & =2\left(x^{2}-1\right), \\
G(x) & =2 \mu((\alpha+\beta) x+\beta-\alpha) .
\end{aligned}
$$

From (31) and (38), we obtain the following system

$$
\left\{\begin{array}{l}
(\alpha+\beta-1)(\alpha+\beta) \mu=2 \\
(\alpha+\beta-1)(\alpha-\beta) \mu=0 \\
\left((\alpha-\beta)^{2}-(\alpha+\beta)\right) \mu=-2 .
\end{array}\right.
$$

Then $\alpha=\beta=\mu=1$. Hence, (38) and (39) becomes $F(x)=x^{2}-1, G(x)=4 x$ and $A(x)=2\left(x^{2}-1\right)$. According to Lemma 3.3(ii), we get

$$
\left(x^{2}-1\right) Q_{n}^{\prime \prime}(x)+4 x Q_{n}^{\prime}(x)+2 Q_{n}(x)=\rho_{n} P_{n}(x), n \geq 0,
$$

where $\rho_{n}=(n+1)(n+2), n \geq 0$.

For $n=1$ in (40), we obtain $\beta_{0}=0$. Given $6=\rho_{1}=6 \lambda_{1} \gamma_{1}^{-1}$, we have $\gamma_{1}=\lambda_{1}$. From Table $\left(\mathbf{C}_{4}\right)$ and by virtue of Lemma 3.2(i), we finally get $\beta_{n}=\xi_{n}=0, n \geq 0$, and $\gamma_{n}=\lambda_{n}=$ $\frac{n(n+2)}{(2 n+1)(2 n+3)}, n \geq 1$. This implies that, $P_{n}(x)=Q_{n}(x)=P_{n}^{(1,1)}(x), n \geq 0$, and then $u_{0}=v_{0}$. In addition, by (13) with $n=1,(14)$ and (15), we get $c=0$. Using Lemma 3.3(i), we finally get $u_{0}=0$, which contradicts the quasi-definiteness of $u_{0}$.

Remark 3.1 : By applying the standard derivative in (29) (resp. (36)) and using (2.22) and (2.23) of page 149 of Chihara's book [12], we get (38) and (46) of the reference [3], by the fact that $\mathscr{O}_{c ; 1,3,2}=D T_{c}$.

\section{ACKNOWLEDGEMENT}

The authors would like to thank the referees for their corrections and many valuable suggestions. The authors extend their appreciation to the Deanship of Scientific Research at Majmaah University for funding this work under Project Number No. (RGP-2019-5). 


\section{REFERENCES}

1. F. Abdelkarim and P. Maroni, The $D_{w}$-classical orthogonal polynomials, Result. Math., 32 (1997), 1-28.

2. B. Aloui, F. Marcellán and R. Sfaxi, Classical orthogonal polynomials with respect to a lowering operator generalizing the Laguerre operator, Integral Transforms Spec. Funct., 24(8) (2013), 636-648.

3. B. Aloui, Hahn's problem with respect to a third-order differential operator, Transylv. J. Math. Mech., 6(2) (2014), 85-100.

4. B. Aloui, Characterization of Laguerre polynomials as orthogonal polynomials connected by the Laguerre degree raising operator, Ramanujan J., 45(2) (2018), 475-481.

5. W. Al-Salam, Characterization theorems for orthogonal polynomials, In: Orthogonal Polynomials: Theory and Practice, P. Nevai Editor, NATO ASI Series C, Vol. 294. Kluwer Acad. Publ., Dordrecht, (1990), $1-24$.

6. W. Al-Salam and T. S. Chihara, Another characterization of the classical orthogonal polynomials, SIAM J. Math. Anal., 3 (1972), 65-70.

7. I. Area, A. Godoy, A. Ronveaux and A. Zarzo, Classical symmetric orthogonal polynomials of a discrete variable, Integral Transforms Spec. Funct., 15 (2004), 1-12.

8. N. M. Atakishiyev, M. Rahman, and S. K. Suslov, On classical orthogonal polynomials, Constructive Approx., 11 (1995), 181-223.

9. Y. B. Cheikh and M. Gaied, Characterization of the Dunkl-classical symmetric orthogonal polynomials, Appl. Math. Comput., 187 (2007), 105-114.

10. S. Bochner, Über sturm-Liouvillesche polynomsysteme, Math Z., 29 (1929), 730-736.

11. A. Branquinho, J. Petronilho, and F. Marcellán, Classical orthogonal polynomials, a functional approach, Acta Appl. Math., 34 (1994), 283-303.

12. T. S. Chihara, An introduction to orthogonal polynomials, Gordon and Breach, New York, 1978.

13. E. Godoy, I. Area, A. Ronveaux, and A. Zarzo, Minimal recurrence relations for connection coefficients between classical orthogonal polynomials: continuous case, J. Comput. Appl. Math., 84 (1997), 257275.

14. E. Grosswald, Bessel Polynomials, (Lecture Notes in Mathematics 698) Berlin: Springer, 1978.

15. V. W. Hahn, Über die Jacobischen polynome und zwei verwandte polynomklassen, Math. Z., 39 (1935), 634-638.

16. V. W. Hahn, Über Orthogonalpolynome, die q-Differenzengleichungen genügen, Math. Nach., 2 (1949), 4-34. 
17. L. Khériji and P. Maroni, The $H_{q}$-classical orthogonal polynomialsm Acta. Appl. Math., 71 (2002), 49-115.

18. K. H. Kwon and G. J. Yoon, Generalized Hahn's theorem, J. Comput. Appl. Math., 116 (2000), 243-262.

19. N. N. Lebedev, Special functions and their applications, (Revised English Edition, Translated and Edited by Richard Silverman 1965), Dover Publications New York, 1972.

20. A. F. Loureiro and P. Maroni, Quadratic decomposition of Appell sequences, Expo. Math., 26 (2008), 177-186.

21. P. Maroni, Une théorie algébrique des polynômes orthogonaux Applications aux polynômes orthogonaux semi-classiques, In Orthogonal Polynomials and their Applications, C. Brezinski et al., Editors, IMACS Ann. Comput. Appl. Math., 9 (1991), 95-130.

22. P. Maroni, Variations around classical orthogonal polynomials. Connected problems, J. Comput. Appl. Math., 48 (1993), 133-155.

23. P. Maroni, Fonctions Eulériennes, Polynômes Orthogonaux Classiques. Techniques de l'Ingénieur, Traité Généralités (Sciences Fondamentales) A 154 Paris. (1994), 1-30.

24. A. F. Nikiforov, S. K. Suslov, and V. B. Uvarov, Classical orthogonal polynomials of a discrete variable, Springer Series in Computational Physics, Springer, Berlin, 1991.

25. A. Zarzo, I. Area, E. Godoy, and A. Ronveaux, Inversion problems for classical continuous and discrete orthogonal polynomials, J. Phys. A: Math. Gen., 30 (1997), L35-L40. 